\title{
La autoestima, la autonomía y el apoyo a las necesidades psicológicas básicas en personas con discapacidad visual
}

\begin{abstract}
Self-esteem, autonomy and support for basic psychological needs in visually impaired people
\end{abstract}

Julio Alfonso Mocha Bonilla. ${ }^{1}$, Yury Rosales Ricardo. ${ }^{2}$, Carmen Dolores Chávez Fuente. ${ }^{3}$ \& Ximena Cumandá Miranda López. ${ }^{4}$

Recibido: 10-02-2019 / Revisado: 15-02-2019 /Aceptado: 04-03-2019/ Publicado: 14-06-2019

\begin{abstract}
.
DOI: https://doi.org/10.33262/cienciadigital.v3i2.5.536

The social and sporting inclusion of people with blindness is a topical issue in the world of sport. The aim of this study was to analyses self-esteem, autonomy and support for basic psychological needs in sport. For this purpose, a quasi-experimental methodology was designed, with a pre and posttest study. It was applied in Canton Ambato and was carried out with 40 participants with total visual disability, using a differentiated sports training (EDD). A sports plan was presented to work with people with blindness, applying training loads, the training loads present different demands according to the psychic possibilities of the athlete, based on the above, the intervention and planning of differentiated training was structured on the basis of two well-defined aspects: firstly, differentiated sports training and secondly the psychological aspect. Those

\footnotetext{
${ }^{1}$ Universidad Técnica de Ambato, Facultad de Ciencias Humanas y de la Educación. Unidad de Investigación. Carrera de Cultura Física. Pedagogía de la Actividad Física y Deporte. Ambato, Ecuador. ja.mocha@uta.edu.ec

${ }^{2}$ Universidad Técnica de Ambato, Facultad de Ciencias Humanas y de la Educación, Carrera de Cultura FísicaPedagogía de la Actividad Física y Deporte. Ambato, Ecuador. y.rosales@uta.edu.ec

${ }^{3}$ Universidad Técnica de Ambato, Facultad de Ciencias Humanas y de la Educación, Carrera de Educación Básica. Ambato, Ecuador. cd.chavez@uta.edu.ec

4 Universidad Técnica de Ambato, Facultad de Ciencias Humanas y de la Educación, Carrera de Psicopedagogía. Ambato, Ecuador.xc.miranda@uta.edu.ec
} 
involved were submitted to the model of sports-psychological intervention, which lasted 11 months. All analyses were carried out using the applications of the statistical package SPSS 22. The results obtained in the experimental group show significant improvements in the scores of the three indicators, it is concluded that the psychological effects can be perceived in a mediate way. In this sense, to the extent that the person with blindness perceives that he or she can perform new physical exercises autonomously, without the need for a sports guide, he or she has a greater perception of competence and autonomy in the sports field.

Keywords: Intervention, Self-esteem, Autonomy, Basic Psychological Needs, Differentiated Training.

\section{Resumen.}

La inclusión social y deportiva de personas que presentan ceguera es un tema de total actualidad en el mundo del deporte. El objetivo del presente estudio fue analizar la autoestima, la autonomía y el apoyo a las necesidades psicológicas básicas en el deporte. Para ello se diseñó una metodología cuasi-experimental, con un estudio pre y post test. Se aplicó en el Cantón Ambato y se realizó con 40 participantes que presentan discapacidad visual total, utilizando un entrenamiento deportivo diferenciado (EDD). Se presentó una planificación deportiva para trabajar con personas con ceguera, aplicando cargas de entrenamiento, las cargas de entrenamiento presentan diferentes exigencias de acuerdo a las posibilidades psíquicas del deportista, en base a lo expuesto la intervención y planificación del entrenamiento diferenciado fue estructurado en base a dos aspectos bien definidos: en primer lugar, el entrenamiento deportivo diferenciado y en segundo lugar el aspecto psicológico. Se sometió a los involucrados al modelo de intervención deportivo-psicológico, el cual tuvo una duración de 11 meses. Todos los análisis fueron realizados utilizando las aplicaciones del paquete estadístico SPSS 22. Los resultados obtenidos en el grupo experimental muestran mejoras significativas en las puntaciones de los tres indicadores, se concluye que los efectos psicológicos pueden percibirse de forma mediata. En este sentido, en la medida que la persona con ceguera percibe que puede realizar ejercicios físicos nuevos, de forma autónoma, sin necesidad del guía deportivo, tiene mayor percepción de la competencia y la autonomía en el campo deportivo.

Palabras claves: Intervención, Autoestima, Autonomía, Necesidades Psicológicas Básicas, Entrenamiento diferenciado. 


\section{Introducción.}

La Organización Mundial de la Salud, argumentó la importancia de un modelo inclusivo, valorando los derechos humanos y fomentando una verdadera inclusión en función de programas de ejecución e intervención (OMS, 1994), mientras que en el 2001 se publicó la clasificación internacional del funcionamiento de la discapacidad y salud, la cual menciona el derecho e igualdad de oportunidades y la integración social, posteriormente la Organización Panamericana de la Salud en octubre del 2014 conjuntamente con los miembros de la región de las Américas aprobaron el plan de acción para la prevención de la ceguera y las deficiencias visuales (OPS, 2014), siendo esto el punto de partida para empezar el trabajo de investigación.

Al hablar de discapacidades Minaire (2012), enuncia algunos modelos de intervención en el marco de la rehabilitación de las personas con discapacidad, con lo cual se han logrado grandes aportes en el campo biomédico, discapacidad situacional y mejora de la calidad de vida (Pierre, 1992), dentro del aspecto biomédico se ha incorpora algunas variables como el diagnóstico, los síntomas y los factores psicológicos, mientras que en el campo situacional y de calidad de vida se presenta la integración social al medio y la supresión de barreras sociales que limitan a las personas con discapacidad dentro del ambiente físico, social y actitudinal (Santana Cansado, 2013).

La Ley sobre Igualdad de Oportunidades e Inclusión Social de Personas con Discapacidad en el año 2010, asume como objetivo "asegurar el derecho a la igualdad de oportunidades de las personas con discapacidad, con el fin de obtener su plena inclusión social, asegurando el disfrute de sus derechos y eliminando cualquier forma de discriminación fundada en la discapacidad". Al hablar de discapacidad visual (DV) Vallejo Agudelo (2016) menciona que es un término general que relaciona las deficiencias funcionales y/o estructurales del sistema visual, con las limitaciones en las actividades y la restricción en la participación de una persona, de esta manera se toma como parámetro la capacidad real funcional y las restricciones que el déficit visual genere en su desempeño.

Por su parte Céspedes (2005) afirma que en los últimos años la atención en rehabilitación a las personas con discapacidad se ha contextualizado en un concepto integrador sobre las actuaciones sociales dirigido a garantizar la igualdad de oportunidades. De lo expuesto anteriormente se puede manifestar que el mundo de la discapacidad ha recibido una 
significativa atención profesional y social en los últimos años; los problemas, dificultades y barreras que encuentran las personas con discapacidad visual han pasado a ser expuestos y analizados públicamente, cada vez es mayor el número de personas que se incorpora a la tarea de mejorar las condiciones de vida y el bienestar de las personas que presentan ceguera. La normativa legal en cada país mediante su Constitución Vigente especifican la no discriminación de personas que presenten discapacidad (Garcia, 2006). En el Ecuador las políticas gubernamentales han asumido el tema de la discapacidad desde una perspectiva integral, cuyo propósito es lograr una verdadera inclusión social de las personas que presentan ceguera, es decir, una verdadera cultura de respeto a sus derechos desde todos los ámbitos: productivo, económico, educativo, cultural y deportivo.

El Gobierno Nacional de Ecuador en el año 2013 empezó a dar cumplimiento a la igualdad de oportunidades y a mejorar la calidad de vida de la población a través del Sistema Nacional de Inclusión y Equidad Social, el cual tiene como objetivo "Fomentar el tiempo dedicado al uso del tiempo libre en actividades físicas, deportivas y otras que contribuyan a mejorar las condiciones físicas, intelectuales y sociales de la población" (Consejo Nacional de Planificacion, 2013), enmarcado en la Constitución Política del Ecuador, sección sexta, en su Art. 381.- "El Estado protegerá, promoverá y coordinará la cultura física que comprende el deporte, la educación física y la recreación, (...) y fomentará la participación de las personas con discapacidad (Asamblea Constituyente Republica del Ecuador, 2011). En función de la normativa legal expuesta, se persigue un fin social, una integración sociodeportiva mediante la práctica del deporte, ya que numerosas investigaciones han constatado que la práctica del deporte provoca en las personas con discapacidad mejoras físicas y beneficios psicológicos como lo afirma el padre de la psicología deportiva Coleman Griffith (1925). En base de lo mencionado el objetivo del estudio fue analizar: la autoestima, la autonomía y el apoyo a las necesidades psicológicas básicas en el deporte.

De acuerdo a la Teoría de la Autodeterminación (TAD) las Necesidades Psicológicas Básicas son definidas como algo innato, universal y esencial para la salud y el bienestar, constituyen un aspecto natural en los seres humanos y se aplican a todas las personas, sin tener en cuenta el género, grupo o cultura (Sánchez, 2017); por lo tanto las necesidades psicológicas básicas son necesarias en la vida personal, las cuales se clasifican en tres autonomía, competencia y relación social, cada una está relacionada con el bienestar psicológico del atleta, de faltar 
alguna de estas necesidades el deportista no podría realizar la actividad deportiva de manera óptima (López-Wallea M, 2017), de lo expuesto se enfatiza la existencia de tres necesidades psicológicas básicas esenciales para el crecimiento psicológico, el desarrollo social y el bienestar de la persona: autonomía la cual es la regulador de la propia conducta; competencia conocida como la necesidad de producir los resultados deseados (maestría deportiva) y la relación que hace referencia a relacionarse con los demás y con el contexto social (Fernández-Río, 2017) .

Varios estudio apoyan la utilización de la escala de las necesidades psicológicas: Al analizar una unidad didáctica de juegos cooperativos en función para de la motivación auto determinada, las necesidades psicológicas básicas y el disfrute en escolares de educación primaria durante las clases de educación física, los resultados muestran que el programa de juegos pueden incidir de manera positiva (Paton, 2017); en el ámbito deportivo, influye mucho la relación entrenador deportista, donde interactúan entre sí para obtener un crecimiento físico y emocional, basado en la teoría de la autodeterminación la cual analiza las conductas humanas, se concluye que es importante fomentar el desarrollo integral y el bienestar psicológico mediante el apoyo a la autonomía de los deportistas (Pulido González, 2017), mientras que las necesidades psicológicas básicas de motivación, autodeterminación y bienestar en alumnos que practican danza, muestran que es necesario propiciar un ambiente de motivación y confianza entre alumno - profesor, apoyando la autonomía hacia los estudiantes para que puedan desenvolverse de la mejor manera (Garay, 2017).

Es así que al realizar actividades físico-deportivas monitoreadas con profesores especialistas de Educación Física, aplicando la Escala de Satisfacción de Necesidades Psicológicas muestra como resultado la motivación y la satisfacción tanto del estudiante como del docente, lo cual influye en el comportamiento y la motivación (Orozco, 2018). El contexto deportivo para personas con discapacidad no está exento del apoyo psicológico, por lo tanto la adaptación que tiene el ser humano para adaptarse al medio debe brindar factores físicos y psicológicos, los cuales evolucionan desde movimientos simples y espontáneos hacia acciones motrices más complejas (Rosa, 2018).

Por tanto, el objetivo de este estudio fue analizar la autoestima, la autonomía y el apoyo a las necesidades psicológicas básicas en el deporte. 


\section{Metodologia.}

Se diseñó una metodología cuasi-experimental, con un estudio pre y post test con el fin de fomentar la incluir social en las personas con discapacidad visual. El experimento de tipo cuantitativo fue realizado en el Cantón Ambato y se realizó con 40 participantes que presentan discapacidad visual total. Sujetos a quienes se les aplicó un entrenamiento deportivo diferenciado basado en lo expuesto por Verkhoshansky (2001) el cual manifiesta que la resistencia a menudo se identifica como la capacidad del deportista en ejecutar una carrera de forma prolongada, es decir, un trabajo muscular sin que disminuya su eficacia (Kerkhoshasky, 2002).

De acuerdo a esto se presenta una planificación deportiva para trabajar con personas con ceguera, aplicando cargas de entrenamiento, concordando con Platonov (2001) las cargas de entrenamiento presentan diferentes exigencias de acuerdo a las posibilidades psíquicas del deportista, en base a lo expuesto la intervención y planificación del entrenamiento diferenciado fue estructurado en base a dos aspectos bien definidos: en primer lugar el entrenamiento deportivo diferenciado y en segundo lugar el aspecto psicológico (Platonov, 2001), relacionando estas dos teorías se sometió a los involucrados al modelo de intervención deportivo-psicológico, el cual tuvo una duración de 11 meses.

La descripción de los pasos metodológicos que lleva la experimentación de este estudio se describe a continuación:

\section{Protocolos e Instrumentos}

La operacionalización del método de intervención estuvo estructurada en base a un cronograma establecido en relación a la consecución del objetivo propuesto, aplicando tres instrumentos validados para medir las necesidades psicológicas básicas en el deporte, la autoestima y el apoyo a la autonomía de las personas con ceguera.

El método de intervención se pudo aplicar mediante un programa metodológico de entrenamiento deportivo para las carreras atléticas, realizado mediante la preparación y participación con guías deportivos (entrenamiento diferenciado), con lo cual se pudo analizar el rendimiento deportivo y psicológico de los participantes. 
La aplicación y evaluación del programa se aplicó mediante una metodología cuasi experimental, con un diseño de comparaciones intrasujeto para valorar el impacto de la intervención; durante la experimentación se realizaron chequeos médicos a la población involucrada con el fin de evaluar y detectar posibles riegos o enfermedades antes de iniciar el programa de intervención (EDD), en el grupo control y el grupo experimental antes y después de la intervención se emplearon tres tipos de test para comparar las necesidades psicológicas básicas en el deporte, al final del primer mes se estableció la primera toma (pretest) iniciando con un control médico, la intervención se realizó con normalidad en función de la cargas de entrenamiento personalizado con una duración de nueve meses.

Finalmente en el onceavo mes, se realizó la segunda toma (postest) específicamente para poder medir las necesidades psicológicas básicas en el deporte, la autoestima y el apoyo a la autonomía de las personas con ceguera, para lo cual se utilizaron: La Escala de Satisfacción de las Necesidades Psicológicas en el Deporte (Domínguez, et al. 2010); la Escala de Autoestima de Rosenberg (RSES) (Baños, 2000) y la escala de Percepción de Apoyo a la Autonomía en Contextos Deportivos (EPAAD) (León, 2012).

Estos instrumentos fueron aplicados antes de iniciar la intervención (pretest) y posterior al método de intervención (postest), con lo cual se pretendió verifica el bienestar psicológicodeportivo de las personas con ceguera en el cantón Ambato.

\section{Análisis de Datos}

De un total de 40 sujetos con discapacidad visual, los cuales participaron del método de intervención (programa de entrenamiento deportivo diferenciado), en primer lugar se llevaron a cabo análisis descriptivos para ambos grupos. En segundo lugar, con el fin de comprobar el efecto del programa de intervención, se realizó un análisis general pre o post test. Todos los análisis fueron realizados utilizando las aplicaciones del paquete estadístico SPSS 22.

\section{Resultados.}

En primera instancia se presentan los estadísticos descriptivos analizados en la toma de pre test. 


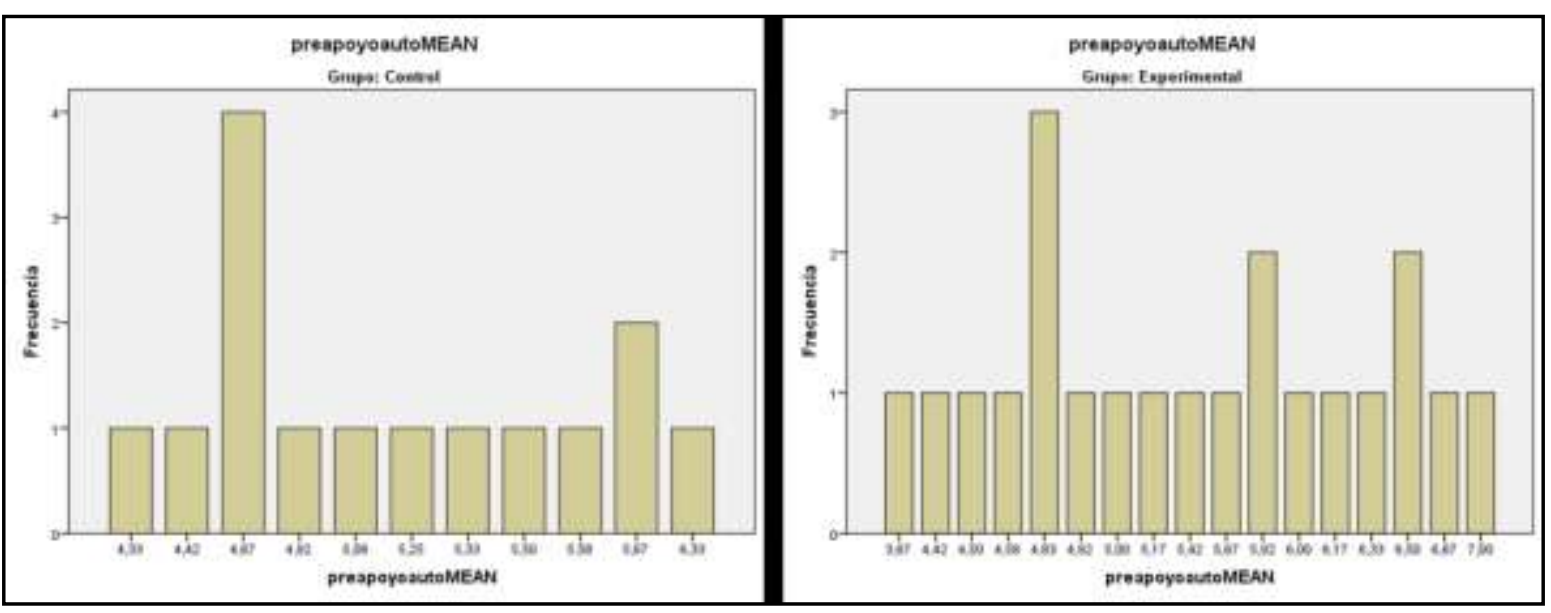

Figura 1. Frecuencias en percepción de apoyo a la autonomía.

Fuente: Elaboración propia.

En la figura 1 respecto a la percepción de apoyo a la autonomía que los deportistas tienen en relación a sus entrenadores, teniendo en cuenta que los valores más bajos en esta variable son de 1 y la más alta 7, observamos que en general los valores se distribuyen en valores que pueden considerar moderados.
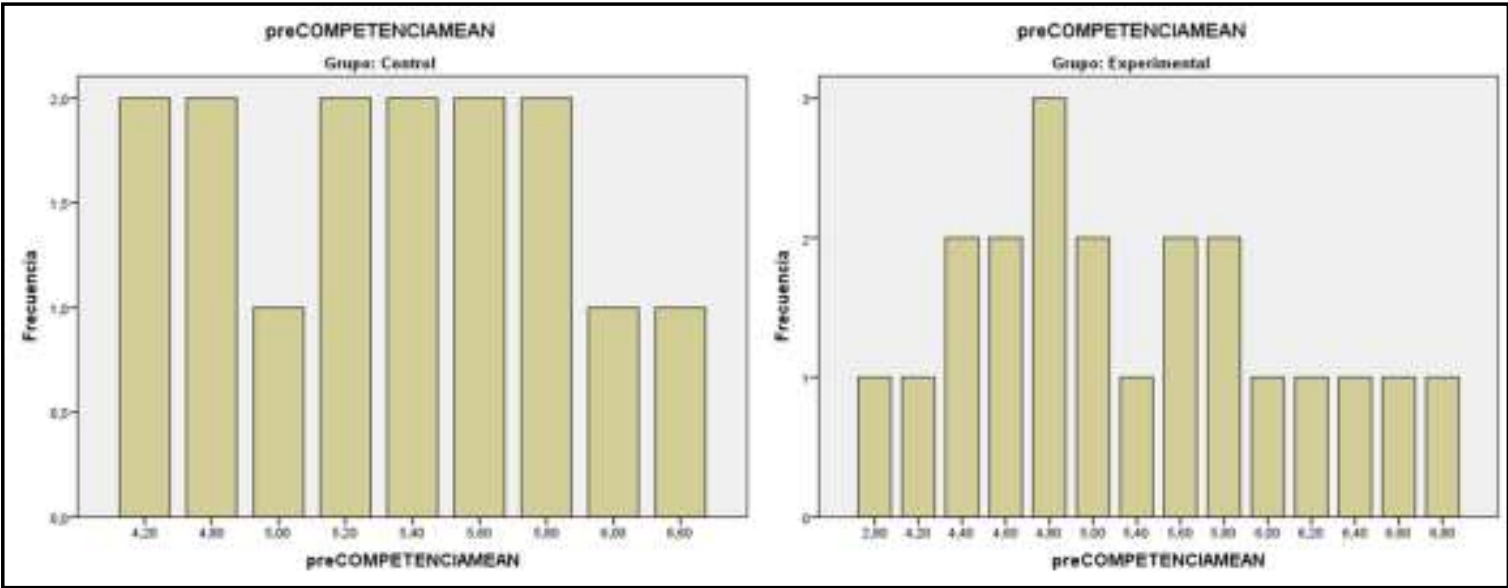

Figura 2. Frecuencia observada en percepción de la competencia.

Fuente: Elaboración propia.

La figura 2 muestra la frecuencia observada en la variable percepción de la competencia. Al igual que en el caso anterior, los valores mínimos y máximo son de 1 y 7 , respectivamente. Como podemos observar, los valores en ambas muestras son moderadamente altos. 


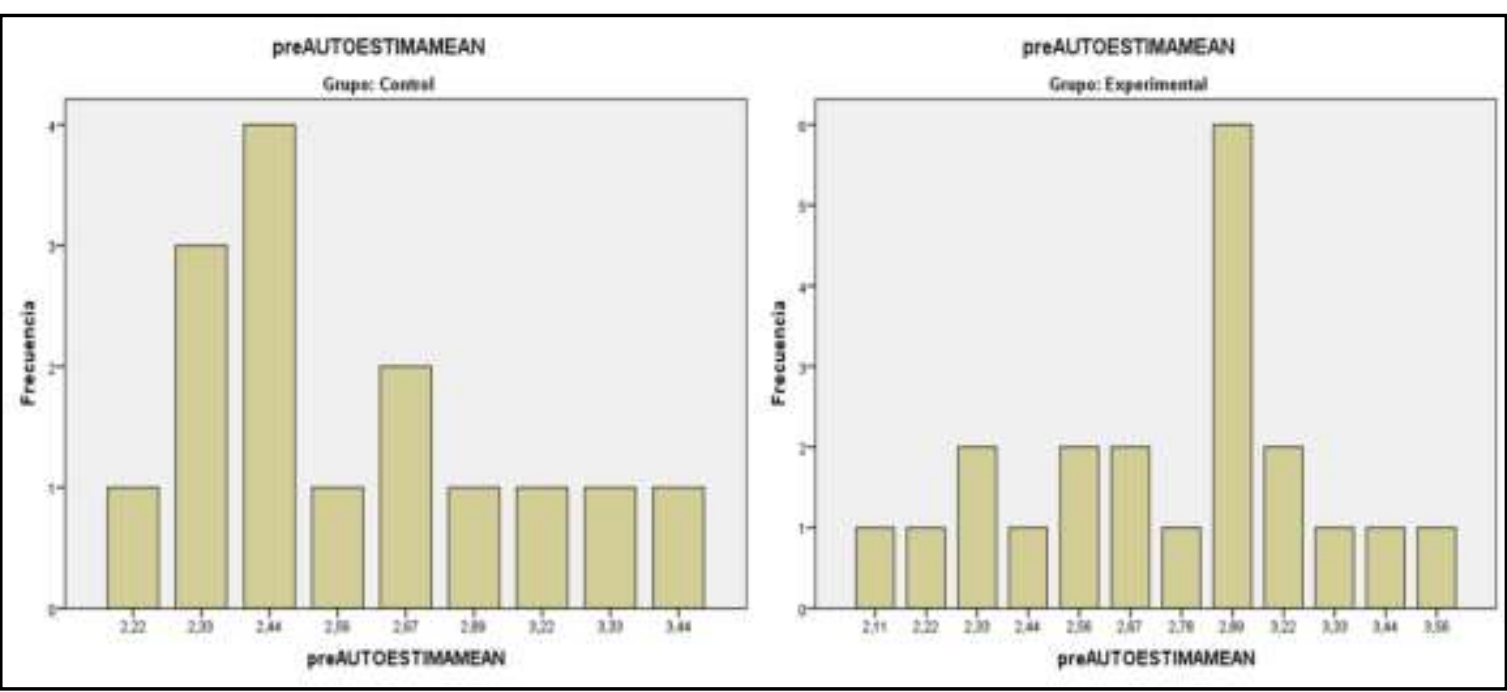

Figura 3. Frecuencias observadas en autoestima.

Fuente: Elaboración propia.

Por último, la figura 3 muestra los niveles de autoestima de los dos grupos antes de la intervención. Es importante señalar que en este caso, las puntuaciones oscilar entre 1 y 4 , donde 4 es el nivel más alto de autoestima. Como podemos observar, tanto en el grupo control como en el experimental, los valores se sitúan en valores medios y moderadamente altos.

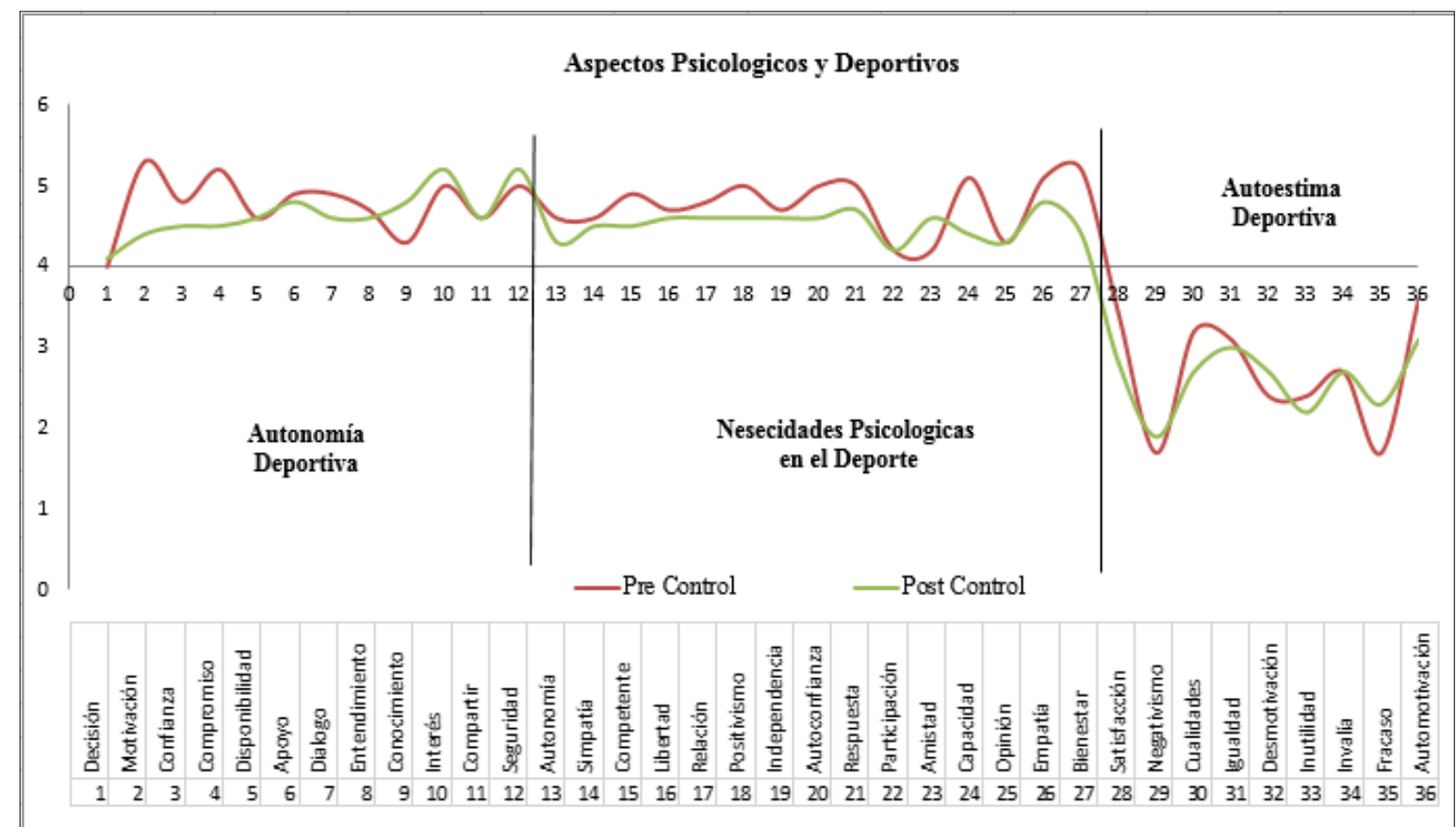

Figura 4. Frecuencias Observadas en los aspectos psicológicos y deportivos pre test grupo control. Fuente: Elaboración propia.

Una vez analizados los 36 factores utilizados para medir los tres aspectos psicológicos y deportivos del grupo control; se puede observar que la autonomía presenta valores del pre 
test promedio de 4,7; mientras que en el post test se obtuvo un valor de 4,6. En relación a las necesidades psicológicas básicas en el deporte se aprecia un valor promedio en el pre test de 4,7; mientras que en el post test se obtuvo un valor de 4,5. Finalmente en el aspecto de autoestima el valor promedio del pre test fue de 2,6 al igual que en el post test.

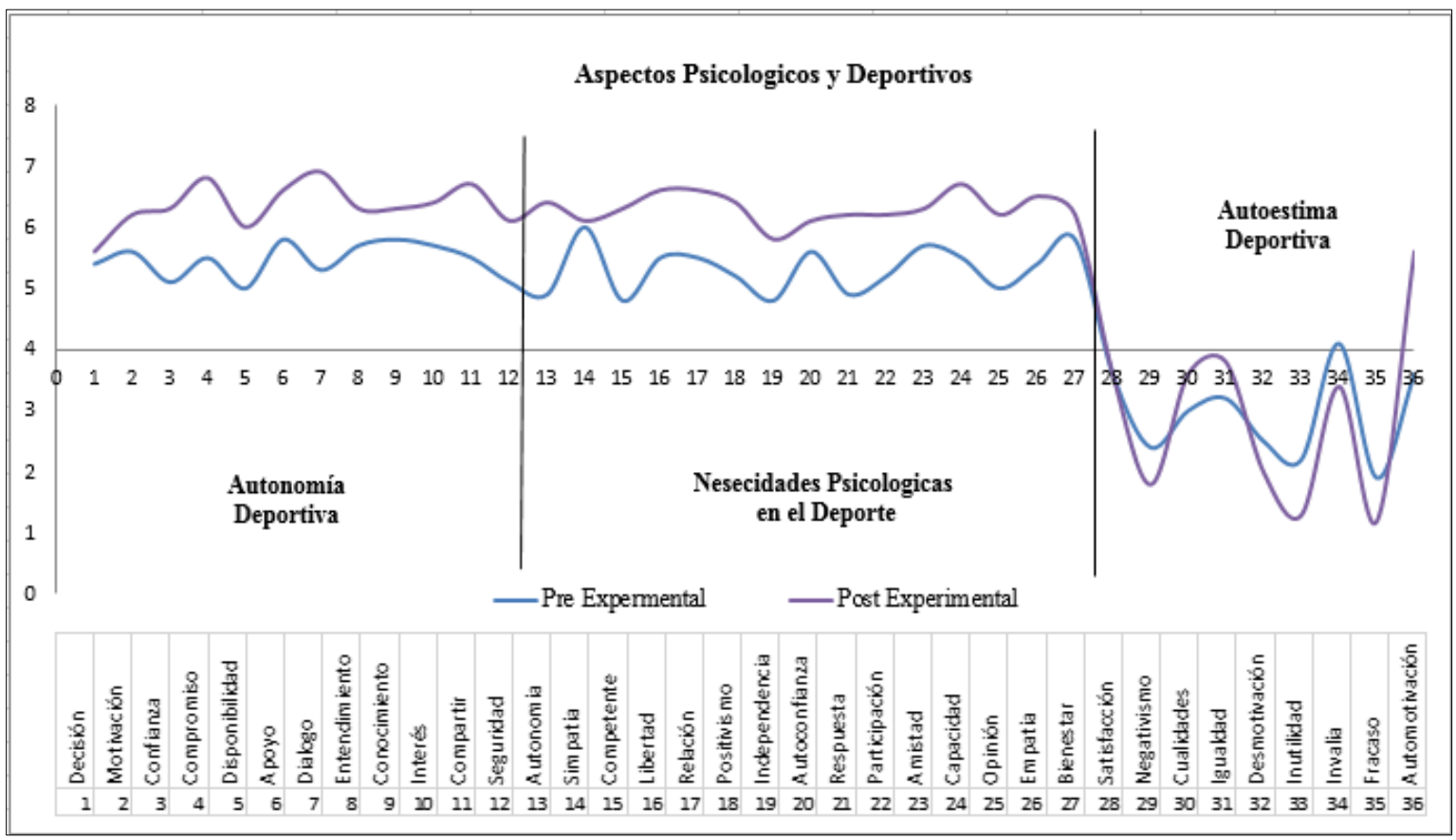

Figura 5. Frecuencias Observadas en los aspectos psicológicos y deportivos post test grupo experimental. Fuente: Elaboración propia.

Luego de la realización de la intervención se aplica los instrumentos, para analizar los 36 factores al grupo experimental; se puede observar que la autonomía presenta valores en el pre test cuyo promedio fue de 5,4; mientras que en el post test se obtuvo una mejora llegando a un valor promedio de 6,3. En relación a las necesidades psicológicas básicas en el deporte se aprecia un valor promedio para el pre test de 5,3; mientras que en el post test se obtuvo una mejoría del valor promedio llegando a 6,3. Finalmente en el aspecto de autoestima el valor promedio del pre test al igual que en el post test fue de 2,9 respectivamente.

Agradecimientos: Queremos expresar nuestro agradecimiento al grupo de deportistas no videntes del Club Fénix de la ciudad de Ambato; a la Carrera de Cultura Física y Pedagogía de la Actividad Física y Deporte por el apoyo para la realización de nuestro trabajo de investigación. 


\section{Conclusiones.}

En el presente estudio se tuvieron en cuenta las variables de carácter psicológico que tienen que ver con los aspectos emocionales y de motivación, los cuales muestran una significativa progresión al aplicar el método de intervención (ED).

Los efectos pueden explicarse debido a que los aspectos psicológicos pueden percibirse de forma mediata. En este sentido, en la medida que la persona con ceguera percibe que puede realizar ejercicios físicos nuevos, de forma autónoma, sin necesidad del guía deportivo, tiene mayor percepción de la competencia y la autonomía. Esta percepción está relacionada directamente con la motivación autónoma, es decir, una mejora en su autoestima personal.

Finalmente, la realización del estudio obtuvo un reconocimiento social, promoviendo una verdadera inclusión socio-deportiva mediante las competencias atléticas, en las cuales las personas con discapacidad visual del cantón Ambato demostraron sus capacidades físicas, autoestima personal y social al involucrarse como sujetos capaces de desenvolverse en la sociedad mediante la práctica del deporte.

\section{Referencias bibliográficas.}

Asamblea Constituyente Republica del Ecuador. (13 de julio de 2011). http://www.oas.org/juridico/pdfs/mesicic4_ecu_const.pdf. Recuperado el 21 de marzo de 2016, de http://www.oas.org/juridico/pdfs/mesicic4_ecu_const.pdf

Baños, R. M. (2000). Psychometric characteristics in normal and social phobic samples for a Spanish version of the Rosenberg Self-Esteem Scale. Psychological reports, 87(1), 269-274.

Céspedes, G. M. (2005). La nueva cultura de la discapacidad y los modelos de rehabilitación. Aquichán Scielo, Aquichán(vol.5 no.1 Bogotá ), 108-113.

Consejo Nacional de Planificacion, R. d. (24 de junio de 2013). www.buenvivir.gob.ec. Recuperado el 21 de marzo de $2016, \quad$ de http://documentos.senplades.gob.ec/Plan\%20Nacional\%20Buen\%20Vivir\%2020132017.pdf

Domínguez, E. M.-A. (2010). Translation and validation of the Spanish version of the "Échelle de Satisfaction des Besoins Psychologiques" in the sports context. The Spanish Journal of Psychology, 13(1), 101. 
Fernández-Río, J. (28 de 02 de 2017). Papel importante del alumnado, necesidades psicológicas básicas. Cuadernos de Psicología del Deporte, 1-44.

Garay, M. G. (2017). Apoyo a la Autonomía, satisfacción de Necesidades Psicológicas Básicas, Motivación Autodeterminada y Bienestar en bailarines adolescentes de una escuela de danza. INFORMACION PSICOLOGICA,(112), 29-43. Obtenido de http://www.informaciopsicologica.info/OJSmottif/index.php/leonardo/article/view/563

Garcia, P. S. (2006). Aproximacion a la realidad de las personas con discapacidad en latinoamérica. Madrid: Colección Cermies.

Kerkhoshasky, Y. (2002). Teoría y Metodología del Entrenamiento Deportivo. Barcelona: Paidotrivo.

León, J. N.-A. (2012). Translation and validation of the Spanish version of the Echelle des Perceptions du Soutien a l'Autonomie en Sport. Universitas Psychologica, 11(1), 165-175.

López-Wallea M, O.-A. L.-M. (2017). Presentación de las tareas, satisfacción de las necesidades psicológicas básicas y bienestar en atletas universitarios. sciencedirect, 13-20.

OMS. (1994). Clasificacion Internacional de deficiencias, discapacidades y minusvalías. Manual de clasificación de las consecuencias de la enfermedad, publicada de acuerdo con la resolución WHA29.35 de la Vigesimonovena Asamblea Mundial de la salud. Madrid.

OPS. (2014). Pan American Health Organization. Plan of Action for the Prevention of Blindness and Visual Impairment. . Pan American Health Organization. Plan of Action Proceedings of the 53rd Directing Council of PAHO, 66th Session of the Regional Committee of WHO for the Americas. Pan American Health Organization. Plan of Action for the Prevention of Blindness and Visual Impairment. Proceedings of the 53rd Directing Council of PAHO, 66th Sessio Washingthon .

Orozco, A. M. (2018). Satisfacción de necesidades psicológicas y orientaciones de meta en profesorado de Educación Física hacia sus alumnos. Retos: nuevas tendencias en educación física, deporte y recreación, 33, 50-53.

Paton, N. (2017). Los juegos cooperativos: incidencia en la motivación, necesidades psicológicas básicas y disfrute en Educación Primaria. Sportis, 3(3), 589-604.

Pierre, M. (1992). Disease, illness and health: theoretical models of the disablement process. Bull World Health Organ. Disease, illness and health: theoretical models of the disablement process. Bull World Health Organ.

Platonov, V. (2001). Teoría General del Entrenamiento Deportivo Olímpico. Barcelona: Paidotribo.

Pulido González, J. J.-O.-P.-C. (2017). . Frustración de las necesidades psicológicas, motivación y burnout en entrenadores: Incidencia de la formación. Revista de psicología del deporte, 26(1), $26-36$. 
Rosa, G. A. (2018). EmásF: revista digital de educación física. revista digital de educación fisica., $105-124$.

Sánchez, J. M. (2017). Análisis preliminar de las propiedades psicométricas de la versión española de la Escala de Necesidades Psicológicas Básicas en el Ejercicio Físico. . Revista Iberoamericana de Psicología del Ejercicio y el Deporte.

Santana Cansado, P. \&. (2013). ACTITUDES HACIA LA DISCAPACIDAD E INTERVENCIÓN DOCENTE DESDE EL DEPORTE ADAPTADO THE ATTITUDE TOWARDS DISABILITY AND TEACHING INTERVENTION IN ADAPTED SPORTS. Rev.int.med.cienc.act.fís.deporte- vol. 13 - número 49 - ISSN: 1577-0354, vol. 13 - número 49 - ISSN: 1577-0354(número 49 - ISSN: 1577-0354), 17.

Vallejo Agudelo, E. O.-E. (2016). Anatomic review of visual neuroscience. Case report: neurological visual impairment after TBI with chronic subdural hematomas sub bilateral parieto occipital. Revista Mexicana de Oftalmología El seiver, Volume 90,( Issue 1, January-February 2016), Pages 33-42. 


\section{PARA CITAR EL ARTÍCULO INDEXADO.}

Mocha Bonilla, J., Rosales Ricardo, Y., Chávez Fuente, C., \& Miranda López, X. (2019). La autoestima, la autonomía y el apoyo a las necesidades psicológicas básicas en personas con

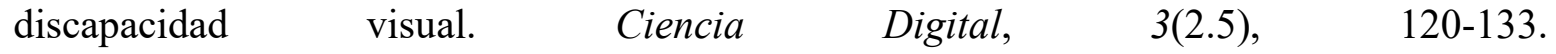
https://doi.org/10.33262/cienciadigital.v3i2.5.536

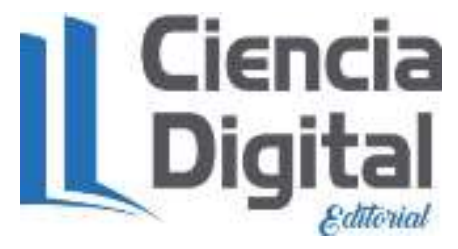

El artículo que se publica es de exclusiva responsabilidad de los autores y no necesariamente reflejan el pensamiento de la Revista Ciencia Digital.

El artículo queda en propiedad de la revista y, por tanto, su publicación parcial y/o total en otro medio tiene que ser autorizado por el director de la Revista Ciencia Digital.
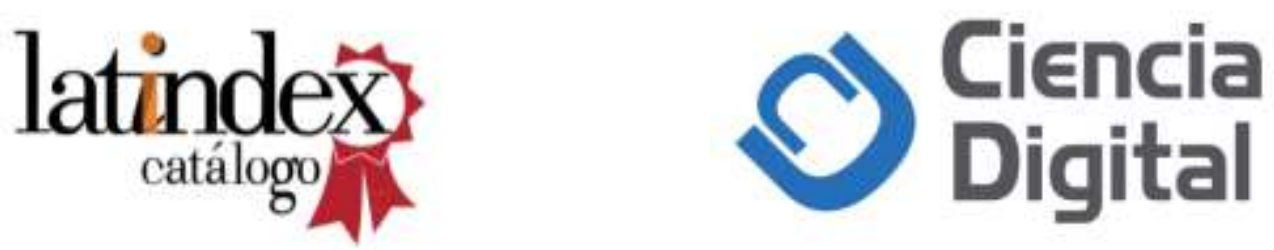